LBNL-54084 Ext

\title{
Intense Attosecond Radiation from an X-Ray FEL - Extended Version*
}

\author{
Alexander A. Zholents and William M. Fawley \\ Lawrence Berkeley National Laboratory \\ University of California, \\ Berkeley, California 94720
}

December 2003

* This work was supported by the Director, Office of Science, Office of High Energy and Nuclear Physics, High Energy Physics Division, of the U. S. Department of Energy, under Contract No. DE-AC03-76SF00098. 
Intense Attosecond Radiation from an X-Ray FEL - Extended Version*

\author{
Alexander A. Zholents and William M. Fawley \\ Lawrence Berkeley National Laboratory, Berkeley, California 94720-8211
}

(Dated: December 1, 2003)

\begin{abstract}
We propose the use of a ultra-relativistic electron beam interacting with a few-cycle, intense laser pulse and an intense pulse of the coherent x-rays to produce a multi-MW intensity, $\mathrm{x}$-ray pulses $\approx 100$ attoseconds in duration. Due to a naturally-occurring frequency chirp, these pulses can be further temporally compressed.

PACS numbers: 41.50.+h, 41.60.Cr, 42.55.Vc, 52.59.-f
\end{abstract}

The ability to study ultrafast phenomena has been recently advanced by the demonstrated production and measurement of a single, 650-attosecond $\left(10^{-18} \mathrm{sec}\right)$, soft x-ray pulse[1]. This has made possible the first pump-probe experiments where the temporal evolution of inner-shell atomic processes was measured[2]. All of these extraordinary results utilized the new availability of intense, few-cycle laser pulses (see [3] and references therein) with a stabilized carrier-envelope phase [4, 5].

The next frontier will be production of attosecond $\mathrm{x}$ ray pulses at even shorter wavelengths than presently demonstrated. Free-electron lasers (FEL's) based upon the principle of self-amplified spontaneous emission (SASE) have recently been considered[6] as a possible source for obtaining sub-fs, short-wavelength x-ray pulses. However, the output radiation of a SASE FEL has quite limited temporal coherence and is composed of many isolated, sub-fs "spikes" whose arrival time is random on a shot-to-shot basis. This may preclude the straight-forward use of SASE FEL's in pump-probe experiments at the attosecond time scale.

In this paper we propose another method involving the radiation of an isolated, attosecond duration, shortwavelength x-ray pulse by electrons selected by their previous interaction with a few-cycle, intense laser pulse. We call this process "seeded attosecond x-ray radiation". In principle it allows excellent temporal synchronization between the attosecond x-ray probe and a pump source that can be the same few-cycle pulse or another signal derived from it. Notably, we show that it is possible to generate a 1-nm x-ray pulse of $\sim 100$-attosecond FWHM duration, which is only $\sim 1 / 25$ th of a single cycle of an optical pump laser. Thus, it is conceivable, for example, to track the temporal evolution of atomic or molecular states during a single optical cycle in the process of laser-assisted photoionization.

Our proposed method requires an ultra-relativistic electron beam, a few-cycle, intense optical laser pulse and an intense pulse of the coherent x-ray radiation, together with a number of magnetic undulators and transport elements. Figure 1 schematically shows how all these components are used to generate the attosecond x-ray pulse.

\footnotetext{
${ }^{*}$ This is the original version of the paper submitted to PRL
}

On the left is a source producing $\sim 100-\mathrm{fs}, \sim 100-\mathrm{MW}$ peak power, x-ray pulses. While such sources do not exist today, studies of SASE FEL's[7] and harmonic cascade FEL's (HC FEL) [8] have suggested approaches which are feasible in principle. As a specific example we choose $2 \mathrm{~nm}$ as the x-ray source wavelength to eventually produce 1-nm wavelength attosecond radiation. However, as long as an intense, coherent source is available, attosecond pulse generation at both longer and shorter wavelengths is also possible with the same scheme.

We have chosen a model HC FEL[9] as the coherent $\mathrm{x}$-ray source. It can be configured such that only part of the electron bunch is used for the x-ray generation, thus leaving another part near the bunch head whose instantaneous energy spread $\sigma_{E}$ has not been degraded by previous FEL interaction in the upstream cascade or even by SASE gain - an advantage not easily realized with a SASE FEL source. The existence of these "virgin" electrons can be ensured by an electron beam pulse duration sufficiently long $(\geq 2 \mathrm{ps}$ ) to account both for jitter in the arrival time of the electron bunch at the $\mathrm{HC}$ FEL entrance (relative to the arrival time of the original seeding laser pulse) and for FEL action in each cascade stage effectively degrading the electron beam quality (by strongly increasing $\left.\sigma_{E}\right)$ in a small potion $(\sim 100 \mathrm{fs})$ of the electron pulse.

After exiting the HC FEL, an achromatic bend inserts the electrons into a two-period wiggler magnet "800-nm modulator". Simultaneously, an 800-nm wavelength, $\sim 1 \mathrm{~mJ}, 5$-fs laser pulse enters this wiggler and co-propagates with the electrons. The technical feasibility of such optical pulses has already been proven[10]. The relative timing between the arrival of the electron beam and the optical pulse is set such that the latter temporally overlaps "virgin" electrons. We presume that the x-ray HC FEL pulse will be seeded with a laser pulse which originates from the same laser source as the fewcycle laser pulse which consequently permits tight synchronization between the two. Since the "virgin" ultrarelativistic electrons and the HC FEL x-ray pulse come from the same electron bunch, one can thus ensure temporal synchronization between each of these three beams.

The carrier-envelope phase of the few-cycle laser pulse is adjusted so that the peak electric field appears at the peak of the envelope when the laser pulse passes the wiggler center. The wiggler's magnetic period and undulator 


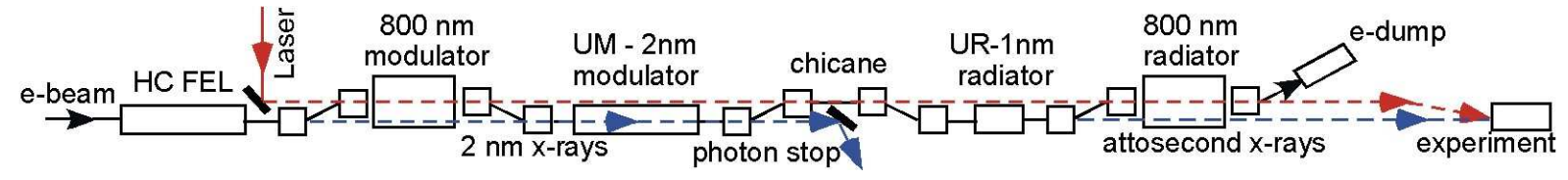

FIG. 1: (Color) A schematic of the components involved in attosecond x-ray pulse production.

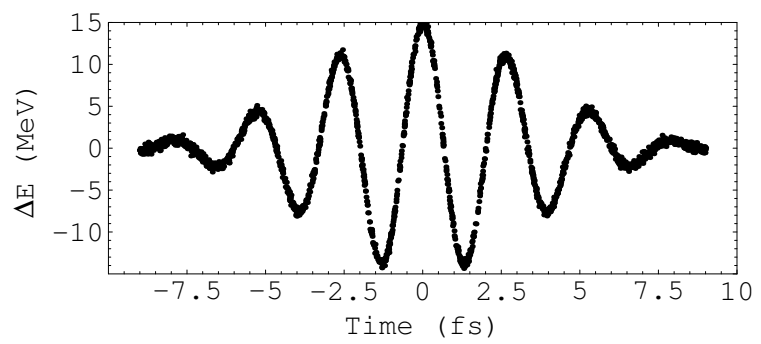

FIG. 2: The calculated energy modulation of the electrons along the electron bunch produced in the interaction with a few-cycle, 800-nm laser pulse in the wiggler magnet presuming an instantaneous electron beam energy spread $\sigma_{E}=0.3 \mathrm{MeV}$.

parameter $K$ are adjusted such that fundamental FEL resonance occurs at the laser wavelength $\lambda_{L}=800 \mathrm{~nm}$. The interaction with the laser light in the wiggler then produces a time-dependent electron energy modulation as shown in Fig. 2. For the laser pulse parameters mentioned above, we expect a central peak energy offset $\Delta \mathcal{E}_{o} \approx 15 \mathrm{MeV}$ which is a factor of 1.35 times larger than those of its nearest neighbors. This relative difference is important when considering the 2-nm energy modulation to be induced in a following undulator.

A second isochronous bend after the wiggler magnet returns the electrons back to the original axis, while the $800-n m$ laser pulse continues to propagate along a parallel, offset path. The electrons now enter a long undulatormodulator (UM) (not shown to scale in Fig. 1), which serves as an energy modulator at 2 -nm wavelength. The coherent, $\geq 100-$ fs long, 2-nm output pulse from the HC FEL co-propagates in the UM with electrons and arrives simultaneously with those electrons that experienced the strong energy modulation at $800 \mathrm{~nm}$. The undulator parameter $K$ of the UM is tuned such that only those electrons very near the peak of the 800-nm energy modulation have the correct energy for resonant FEL interaction with the 2-nm light. The other electrons fall outside the energy bandwidth of the UM FEL and are not significantly modulated. Although the UM is relatively long $\left(L_{u} \approx 5 \mathrm{~m}\right)$, it is shorter than one full FEL gain length so there is little SASE action (which otherwise would produce unwanted microbunching at 2-nm wavelength throughout the 2-ps long electron bunch).

Following [11], the standard 1D FEL particle equations in the zero gain limit may be written as

$$
\frac{d \nu}{d z_{*}}=-\Omega^{2} \sin \theta \quad \text { and } \quad \frac{d \theta}{d z_{*}}=2 \pi \nu
$$

where $z_{*} \equiv z / L_{u}$ is the dimensionless length along the undulator, $\theta$ is the electron phase relative to the FEL ponderomotive well, and $\nu \equiv 2 N_{u}\left(\gamma-\gamma_{R}\right) / \gamma_{R}$ with $N_{u}$ being the number of undulator periods and $\gamma_{R}$ the resonant Lorentz factor. $\Omega$ is the dimensionless, FELequivalent synchrotron tune. Using a perturbation expansion of $\nu$ and $\theta$ in powers of $\Omega^{2}$, one obtains at the undulator end $\left(z_{*}=1\right)$ through order $\Omega^{2}$

$$
\begin{aligned}
& \nu_{f}=\nu_{0}+\frac{\Omega^{2}}{2 \pi \nu_{0}}\left[\cos \left(2 \pi \nu_{0}+\theta_{0}\right)-\cos \theta_{0}\right] \\
& \theta_{f}=\theta_{0}+2 \pi \nu_{0}+\frac{\Omega^{2}}{\nu_{0}}\left[\frac{\sin \left(2 \pi \nu_{0}+\theta_{0}\right)-\sin \theta_{0}}{2 \pi \nu_{0}}-\cos \theta_{0}\right]
\end{aligned}
$$

where $\theta_{0}$ and $\nu_{0}$ refer to values at undulator entrance

$$
\begin{aligned}
\nu_{0}(t) & =\frac{2 N_{u}}{\gamma_{R}}\left[\frac{\Delta \mathcal{E}_{0}}{m c^{2}}\left(\cos \frac{2 \pi c}{\lambda_{L}} t-1\right)+\Delta \gamma_{1}\right]+\delta \nu \\
& \equiv \bar{\nu}_{0}(t)+\delta \nu
\end{aligned}
$$

Here $\Delta \gamma_{1}$ is the detuning offset from the FEL resonant energy and $\delta \nu$ is due to instantaneous energy spread. Equation 3 applies close to the peak of the 800-nm energy modulation.

Downstream of the UM the electrons enter a chicane with a time-of-flight parameter $R_{56}=750 \mathrm{~nm}$ which induces strong microbunching at $\lambda_{x}=2$-nm wavelength and at higher harmonics $\lambda_{x} / n$. In the middle of the chicane, the electron beam orbit is separated $\approx 1 \mathrm{~mm}$ transversely from the path of the x-ray light. This permits a photon stop to block all light coming to this point, which is important for obtaining maximum contrast of the attosecond x-ray pulse over the background radiation. The electron phase at the chicane exit then equals

$$
\begin{aligned}
& \theta_{e}=\theta_{0}+C+2 \pi \nu_{0}(\alpha+1)-\frac{\Omega^{2}}{\nu_{0}} \times \\
& {\left[(2 \alpha+1) \sin \pi \nu_{0} \sin \tilde{\theta}-\left(\frac{\sin \pi \nu_{0}}{\pi \nu_{0}}-\cos \pi \nu_{0}\right) \cos \tilde{\theta}\right]}
\end{aligned}
$$

where $C$ is an energy-independent path-length difference term, $\alpha \equiv R_{56} / 2 N_{u} \lambda_{x}$, and $\tilde{\theta} \equiv \theta_{0}+\pi \nu_{0}$. It is convenient to define the complex bunching factor at the $n^{\text {th }}$ harmonic $b_{n} \equiv\left\langle e^{i n \theta_{e}}\right\rangle$ where the averaging takes place over $\theta_{0}$ and $\delta \nu$. Presuming a uniform phase distribution of electrons in $\theta_{0}$, a Gaussian energy distribution in $\delta \nu$ whose rms value $\sigma_{v} \equiv 2 N_{u} \sigma_{E} / \gamma_{R} m c^{2}$, we obtain from Eq. (4) at the chicane exit

$$
b_{n}\left(\bar{\nu}_{0}\right)=J_{n}\left(n \pi \Omega^{2} \chi\left(\pi \bar{\nu}_{0}\right)\right) e^{i n \phi\left(\bar{\nu}_{0}\right)} e^{-\frac{1}{2}\left(n \pi(2 \alpha+1) \sigma_{v}\right)^{2}}
$$




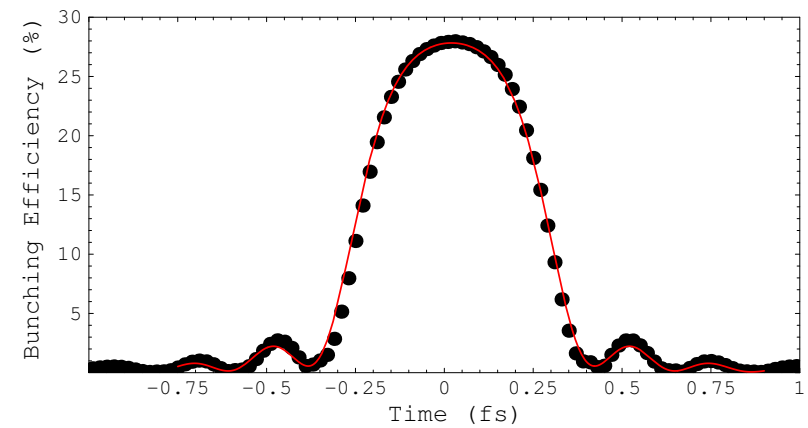

FIG. 3: (Color) Bunching efficiency at 1-nm wavelength versus time along the electron bunch. The solid line shows calculations using Eq.(5) and the dots simulation results from GINGER. The FWHM of the peak is 530 attoseconds.

where $J_{n}$ is the $n^{\text {th }}$-order Bessel function of the first kind,

$$
\chi(x) \equiv \sqrt{\left((2 \alpha+1) \frac{\sin x}{x}\right)^{2}+\frac{1}{x^{2}}\left(\frac{\sin x}{x}-\cos x\right)^{2}}
$$

and $\phi\left(\bar{\nu}_{0}\right)=\pi \bar{\nu}_{0}(2 \alpha+1)+\tan ^{-1}\left[\frac{1}{2 \alpha+1}\left(\frac{1}{\pi \bar{\nu}_{0}}-\frac{1}{\tan \pi \bar{\nu}_{0}}\right)\right]$. Equation (5) is similar to the bunching factor previously obtained in optical klystron theory[12].

In Fig. 3 we plot the bunching amplitude $\left|b_{n}\left(\bar{\nu}_{0}\right)\right|$ for $n=2$ (i.e. 1-nm wavelength) as predicted using Eq. (5) and as calculated by the GINGER simulation code [13]. In both cases we used: $\lambda_{x}=2 \mathrm{~nm}, \lambda_{L}=800 \mathrm{~nm}$, $\gamma_{R} m c^{2}=3 \mathrm{GeV}, \Delta \gamma_{1} m c^{2}=-1.2 \mathrm{MeV}, N_{u}=200$, and $R_{56}=750 \mathrm{~nm}$. For the analytic calculation, we adopted $\Omega^{2}=0.1$. The GINGER simulation presumed an electron beam current of $500 \mathrm{~A}$ with a normalized emittance of $2 \mathrm{~mm}$-mrad, $\sigma_{E}=0.3 \mathrm{MeV}$, and an input x-ray pulse with a temporally constant 150-MW intensity whose electric field Gaussian waist size of $150 \mu \mathrm{m}$ occurred 2-m upstream of the UM entrance. The UM had $K=3.02$ and a $25-\mathrm{mm}$ period.

After the chicane, the electrons proceed to an undulator-radiator (UR) whose function is to produce coherent emission at wavelength $\lambda_{x} / n=1 \mathrm{~nm}$ from the bunched electrons. Here we considered a linearlypolarized UR but note that a helically-polarized UR has an advantage in that no higher harmonics are radiated on axis. To simplify the calculation of the radiation field, we assume that electrons enter the UR as macroparticles (representing microbunches) separated by $1 \mathrm{~nm}$ plus additional displacements caused by the variation of the bunching phase $n \phi\left(\bar{\nu}_{0}\right)$. Due to the short length of the $\mathrm{UR}$, we also neglect any further evolution of the microbunching or FEL gain. Because of the relative longitudinal slippage in the radiator between the electrons and radiation, each macroparticle radiates the $\mathrm{x}$-ray light which has the same number of cycles as the number of periods in the radiator, $N_{R}$. The interference of the waves emitted by all macroparticles defines the output envelope $\tilde{E}(t)$ of the radiation field, $E(t)=\operatorname{Re}\left\{\tilde{E}(t) e^{-i \omega_{x} t}\right\}$,

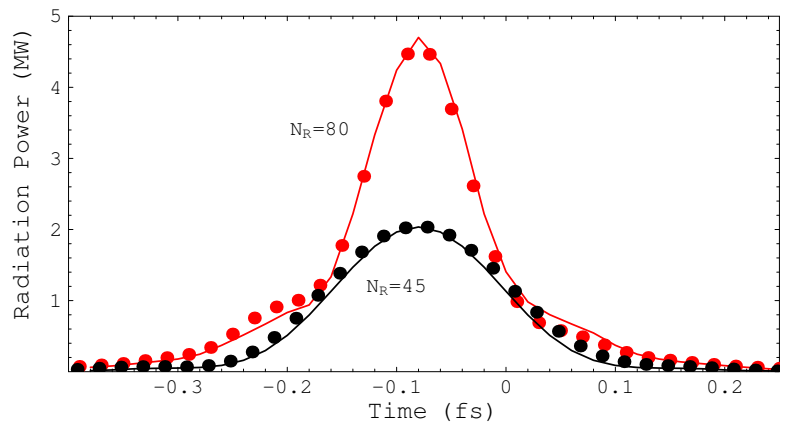

FIG. 4: (Color) Predicted attosecond pulse power at 1-nm wavelength from a radiator with $N_{R}=80$ (top line) and $N_{R}=$ 45 (bottom line) using Eq. (7). Both curves were normalized to the peak intensity of the $N_{R}=80$ simulation results (dots).

where

$$
\tilde{E}(t) \propto \sum_{-\infty}^{\infty} b_{n}\left(\bar{\nu}_{0}\right)_{j} H\left(\frac{N_{R}}{2}-\left|j-\frac{N_{R}}{2}+\frac{c t}{\lambda_{x}}\right|\right)
$$

$\omega_{x} \equiv 2 \pi n c / \lambda_{x}, H(x)$ is the Heaviside function, and $t=0$ corresponds to the time at which the $j=0$ microbunch exits the UR.

The predicted radiation field intensity $I(t) \propto|\tilde{E}(t)|^{2}$ may be determined from Eq. (7). The good agreement shown in Fig. 4 between the analytic predictions for the pulse shape and the GINGER simulation (in which the microbunching and any FEL gain are followed selfconsistently) indicates that the simplifying assumptions for the analytic calculation adopted above appear to be well founded. The rms width $\sigma_{t}$ of the peak is 48 attoseconds for $N_{R}=80$ and 75 attoseconds for $N_{R}=45$, several times shorter than the bunching width structure shown in Fig. 3. We attribute this reduction to slippage effects in the radiator - there is destructive interference (due to temporal variation of bunching phase) occurring between waves emitted by microbunches on opposite sides of the bunching peak. Evidently, for a given temporal bunching structure, the pulse shortening will depend upon the radiator length. In particular, a shorter radiator will result in less destructive interference and thus a longer output pulse. This is confirmed by the calculation for the $N_{R}=45$ case.

There is another interesting phenomenon which is related to slippage and interference effects, namely the variation of the output electric field phase $\Psi$ with time. Figure 5 shows this variation for $N_{R}=45$ calculated using Eq. (7). We fitted the phase calculations by a parabola $\Psi(t)=a\left(t / \sigma_{t}\right)^{2}$ with $a=1.92$. The quadratic component in the temporal phase dependence indicates the presence of a frequency chirp in the output radiation field. This leads to a time-bandwidth product exceeding the ultimate Fourier transform limit by a factor $\sqrt{1+a^{2}}$ [14]. Consequently, the output pulse for the bottom curve in Fig. 4 could potentially be compressed down to $\tilde{\sigma_{t}}=\sigma_{t} / \sqrt{1+a^{2}}=35$ attoseconds. For a longer 


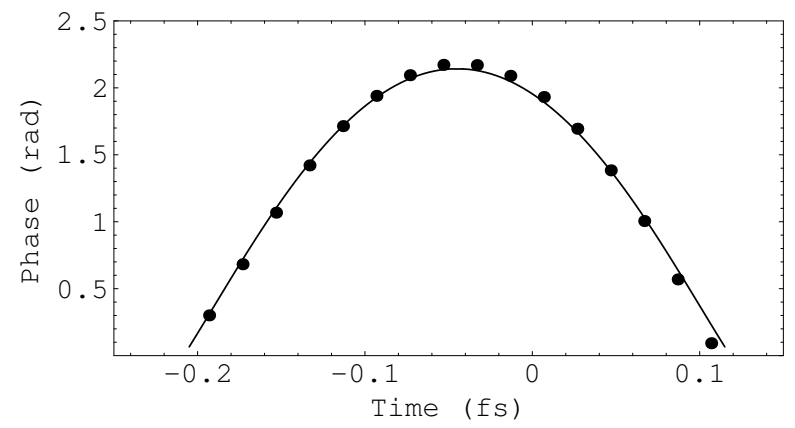

FIG. 5: Predicted attosecond pulse phase at 1-nm wavelength from a radiator with 45 periods. The line shows calculation using Eq. 7 while the dots show GINGER simulation results.

radiator, the frequency chirp lessens and effectively disappears by $N_{R}=80$.

Figure 6 shows the output spectra corresponding to both the coherent attosecond radiation and incoherent spontaneous emission. Due to the relatively large electron beam emittance in our numerical example, the electron beam size in the UR is several times larger than the diffraction-limited size of spontaneous radiation at 1-nm wavelength with the result that the coherent radiation is constrained within a rms solid angle of $\sim 3 \times 10^{-11} \mathrm{sr}$. Spontaneous radiation is emitted into a solid angle approximately two orders of magnitude larger. Therefore, a collimator will help isolate the coherent radiation from the majority of the spontaneous emission. The two spectra are also shifted in wavelength with respect to each other by about $1 \%$. The shift is due to the different energy of microbunched electrons. A double grating monochromator with a path length compensation similar to one addressed in [15] can be additionally used for better selection of the attosecond pulse. Note that by changing the value of the FEL resonant energy (via $K$ ), the 2-nm modulator can microbunch electrons with either a positive or negative $\Delta E$ (see Fig. 2). However, microbunching at positive $\Delta E$ is preferable for a better signal-to-background ratio because then the coherent radiation is shifted to the blue side relative to the peak of spontaneous emission, which has an asymmetrical tail extending to the red side.

In an alternate scenario, we examined the $n=1$ case where both the UM and UR are resonant at 1-nm wavelength. For this case with a presumed input power of $10 \mathrm{MW}$ we needed $N_{u}=400$ to develop sufficient bunching $\left(R_{56}\right.$ remained $\left.750 \mathrm{~nm}\right)$. For a 2-m radiator $\left(N_{R}=80\right)$, the peak output was $\sim 30 \%$ smaller. However, there is an important difference. First, the input power produces small, but non-zero 1-nm wavelength bunching of electrons which lie outside of the $\sim 5-\mathrm{fs}$ region affected by the 800-nm energy modulation, even though these electrons have relatively large energy offsets (i.e. $15 \mathrm{MeV}$ ) from the FEL resonance of the UM. Coherent radiation from these electrons in the radiator produces a narrow spike at exactly $1-n m$ wavelength (i.e. in

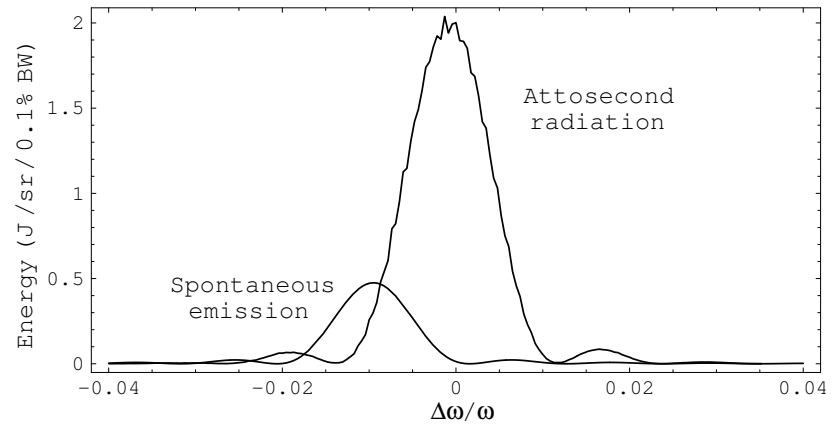

FIG. 6: The predicted on-axis, far-field radiation spectrum $d^{2} P / d \Omega d \omega$ at 1 -nm wavelength from a radiator with 80 periods. The attosecond output was calculated by the GINGER code whereas the spontaneous emission curve is an analytic result for a presumed e-beam pulse duration of $2 \mathrm{ps}$.

the middle of the spectra of the attosecond radiation). This spike is virtually absent for the 2-nm modulation case because the accompanying bunching at 1-nm (arising from non-linear effects) is much smaller.

A key issue for the effective utilization of the attosecond pulse is temporal synchronization with an external laser for pump-probe experiments. We suggest that all components (beginning from the mirror that is used to direct the laser pulse into the $800-\mathrm{nm}$ modulator and ending with the experimental station) should be placed onto the same rigid girder, thus subjecting the various light and electron beam components to the same path length variations (due to thermal expansion and microphonics). An active feedback system will also be needed, including a high-precision path length tracker. We note that to ensure less than 10-attosecond variation in the time-off-flight of electrons from the wiggler magnet to the UR on a pulse-to-pulse basis, the electron beam energy must be kept stable to approximately $5 \times 10^{-5}$ precision and that its entry angle into the $800-\mathrm{nm}$ wiggler magnet should not fluctuate more than few angular beam sizes at this point. Additionally, the magnetic field in each chicane magnet should not fluctuate more than $1 \%$. Fortunately, there is a possible means to determine the relative timing downstream of the UR between the attosecond $\mathrm{x}$-rays and the original, few-cycle 800 -nm laser pulse. Those electrons which underwent the $800-\mathrm{nm}$ energy modulation can, via an achromatic bend following the UR (see Fig. 1) with a relatively large $R_{56}$ coefficient, be strongly bunched at this wavelength. They can then, via a subsequent few-period wiggler magnet, radiate a few-cycle, sub-microjoule pulse of coherent 800-nm emission. This secondary pulse (which is automatically temporally synchronized with the x-ray attosecond pulse) can then be cross-correlated with the original 800-nm modulating pulse to provide a timing signal for accurate pump-probe synchronization.

Presently there exist several proposals to build x-ray facilities based upon harmonic cascade FELs: LBNL LUX[16], BESSY FEL[17], MIT-BATES FEL[18]. The 


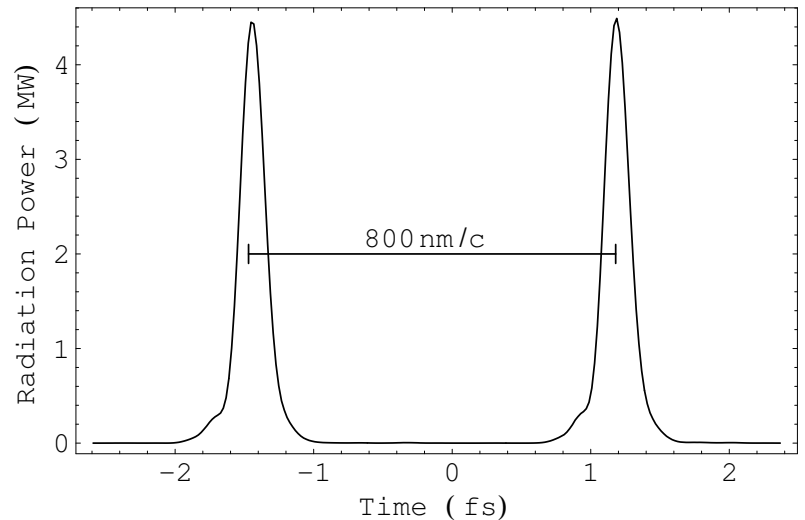

FIG. 7: Predicted instantaneous output power by the GINGER code for the case in which the phase of the intense optical pulse has been shifted 180 degrees (effectively reversing the sign of the energy modulation shown in Fig. 2).

scheme described above for production of attosecond $\mathrm{x}$ ray pulses can be added to any of them with a relatively modest effort as compared to what would be required to build a primary facility. The physical addition of this scheme would not require any other significant change or improvement in the ways these facility will operate. We demonstrated this by purposely choosing for our illustrative example exactly the same electron beam and x-ray light parameters as are currently planned for the LUX facility. Nevertheless, each machine will benefit from adjusting the the attosecond scheme to their needs in particular the choice of the harmonic ratio between the x-ray wavelength for energy modulation and the wavelength for the attosecond radiation. This choice will be largely influenced by the available electron beam energy, emittance, energy spread, and peak current. Moreover, any improvement in these parameters will directly lead to more intense attosecond x-ray pulses.

We may also expect that technological progress in optics will lead to the availability of laser pulses more intense than those considered above. More intense pulses could produce a larger wavelength separation between the attosecond radiation and the spontaneous emission in the radiator, thus improving the contrast of the attosecond pulse. They also might allow one to modify the temporal dependence of the primary energy modulation in the wiggler magnet and obtain more than a single attosecond pulse separated by a short (few femtosecond) time intervals. We point out that one could shift the phase of the laser pulse entering the wiggler magnet by 180 degrees and consequently obtain two separate, attosecond output x-ray pulses separated exactly by one period of the optical cycle as shown in Fig. 7. Similarly, the development of laser sources of intense few-cycle pulses at wavelengths other than $800 \mathrm{~nm}$ will open new opportunities to shape temporally the attosecond x-ray pulses.

Computational resources were provided in part by the National Energy Research Supercomputer Center. This work was supported by the Office of Science, U.S. Dept. of Energy, under Contract No. DE-AC03-76SF00098.
[1] M. Hentchel et al. , Nature, 416, 509 (2001).

[2] M. Drescher et al. , Nature, 419, 803 (2002).

[3] T. Brabec and F. Krausz, Rev. Mod. Phys., 72, 545 (2000).

[4] A. Baltushka et al. , Nature, 421, 611 (2003).

[5] D.J. Jones et al., Science, 288, 635 (2000).

[6] E.L. Saldin, E.A. Schneidmiller, and M.V. Yurkov, Opt. Comm., 212, 377 (2002).

[7] See, e.g., "Linac Coherent Light Source Design Study Report", SLAC-R-521 (1998); "Tesla Technical Design Report", DESY 2001-011 (2001).

[8] L.-H. Yu et al. , Science, 289, 932 (2000).

[9] W. Fawley et al., 2003 Part. Acc. Conf., IEEE 03CH37423, 923 (2003).

[10] S. Sartania et al. , Opt. Lett., 22, 1562 (1997).

[11] W. Colson, Chap. 5 in Laser Handbook, Vol. 6, NorthHolland (1990); J.B. Murphy and C. Pelligrini, Chap. 2, ibid.

[12] N.A. Vinokurov and A.N. Skrinsky, Preprint INP 78-88, Novosibirsk (1978).

[13] W.M. Fawley, LBNL Tech. Rpt. 49625, (2001).

[14] J.-C. Diels and W. Rudolph, Ultrashort Laser Phenomena, Academic Press, (1996).

[15] P. Villoresi, Applied Optics, 38, 6040 (1999).

[16] J.N. Corlett et al. , "A Recirculating Linac-Based Facility for Ultrafast X-ray Science", 2003 Part. Acc. Conf., paper TOAC003 (also LBNL-52605) (2003).

[17] M. Abo-Bakr et al., Nucl. Instr. Meth. A, 483, 470 (2002).

[18] D.E. Moncton and W.S. Graves, "The MIT X-ray Laser Project", Proc. 2003 Synchr. Rad. Instr. Conf., San Francisco (2003). 